\title{
Therapeutic Plasma Exchange as a Treatment for Autoimmune Neurological Disease
}

\author{
Ivana Nieto-Aristizábal, ${ }^{1}$ Álvaro J. Vivas, ${ }^{1,2}$ Pablo Ruiz-Montaño, ${ }^{1,2}$ Cristian C. Aragón, ${ }^{1}$ \\ Iván Posso-Osorio, ${ }^{1}$ Jairo Quiñones, ${ }^{3,4}$ Julián Alejandro Rivillas, ${ }^{2,3}$ and Gabriel J. Tobón (iD ${ }^{1}$ \\ ${ }^{1}$ GIRAT: Grupo de Investigación en Reumatología, Autoinmunidad y Medicina Traslacional, \\ Universidad Icesi and Fundación Valle del Lili, Cra. 98 18-49, Cali, Colombia \\ ${ }^{2}$ Medical School, Universidad Icesi, Calle 18 No. 122-135, Cali, Colombia \\ ${ }^{3}$ Department of Neurology, Fundación Valle del Lili, Cra. 98 18-49, Cali, Colombia \\ ${ }^{4}$ Unit of Neuroimmunology, Fundación Valle del Lili, Cra. 98 18-49, Cali, Colombia \\ Correspondence should be addressed to Gabriel J. Tobón; gtobon1@yahoo.com
}

Received 4 March 2020; Revised 16 June 2020; Accepted 22 June 2020; Published 31 July 2020

Academic Editor: Ricard Cervera

Copyright ( 2020 Ivana Nieto-Aristizábal et al. This is an open access article distributed under the Creative Commons Attribution License, which permits unrestricted use, distribution, and reproduction in any medium, provided the original work is properly cited.

\begin{abstract}
Introduction. Therapeutic plasma exchange (TPE) is commonly used as treatment of certain autoimmune neurological diseases (ANDs), and its main objective is the removal of pathogenic autoantibodies. Our aim was to describe the clinical profile and the experience with the usage of TPE in patients with ANDs at our institution. Methods. This is an observational retrospective study, including medical records of patients with diagnosis of ANDs who received TPE, between 2011 and 2018. Characteristics of TPE, such as number of cycles, type of replacement solution, and adverse effects, were evaluated. The modified Rankin Scale (mRS) was applied to measure the clinical response after the therapy. Results. 187 patients were included with the following diagnoses: myasthenia gravis (MG), $n=70$ (37\%); Guillain-Barré syndrome (GBS), $n=53$ (28.3\%), neuromyelitis optica spectrum disorders (NMOSD), $n=35$ (18.7\%); chronic inflammatory demyelinating polyneuropathy (CIDP), $n=23$ (12.2\%); and autoimmune encephalitis (AE), $n=6(3.2 \%)$. The most used types of replacement solution were albumin $(n=131,70 \%)$ and succinylated gelatin $(n=45,24 \%)$. All patients received a median of five cycles (IQR 5-5). Hypotension and hydroelectrolytic disorders were the main complications. After TPE, 99 patients (52.9\%) showed improvement in the mRS scores and a statistical significance $(p<0.05)$ was seen between the admission score and after TPE for every diagnosis except for CIDP. Conclusion. TPE has an adequate safety profile, and improvement in functionality in treated patients reflects its effectiveness.
\end{abstract}

\section{Introduction}

Therapeutic plasma exchange (TPE) is defined by the American Society for Apheresis (ASFA) 2019 guidelines as "A therapeutic procedure in which the blood of the patient is passed through a medical device which separates plasma from the other components of blood....”. Unlike plasmapheresis, TPE involves plasma removal and replacement with a solution such as a colloid solution (e.g., albumin and/or plasma) or a combination of a crystalloid/colloid solution [1].

Even though it was initially conceived as a treatment for hematological diseases with presumed or demonstrated immune pathophysiology [2], treatment using TPE has been extended to a variety of pathologies including kidney, autoimmune rheumatological, and neurological diseases, with the latter being the pathology most frequently treated by TPE [3]. The increased usage of TPE has likely followed an increased understanding of its mechanisms of action, which range from the removal of pathogenic autoantibodies and immune complexes to improvement in monocyte function [2]. Among the autoimmune neurological diseases (ANDs) treated using TPE, chronic inflammatory demyelinating polyneuropathy (CIDP), Guillain-Barré syndrome (GBS), myasthenia gravis (MG), neuromyelitis optica spectrum 
disorders (NMOSDs), and autoimmune encephalitis (AE) are well described. TPE is typically used alone or in conjunction with other treatment options, such as intravenous immunoglobulins (IVIG) and corticosteroids, as a first-line treatment for some of these disorders.

The great majority of descriptions of the clinical experience of TPE usage for this group of diseases are from North American or European cohorts [4-7]. In addition, environmental and genetic factors differ between populations, and these factors may influence the response the individuals have on different therapeutic interventions. Many of the mentioned ANDs have shown an exponential increase in occurrence in recent years [8-11]. Therefore, it is important to study in depth the safety and effectiveness of their available treatments, such as TPE. In this study, we aimed to describe the factors associated with indications, types of replacement solution, adverse effects, and treatment effectiveness of this intervention in patients with different ANDs.

\section{Material and Methods}

This was a retrospective and observational study conducted at Fundación Valle del Lili, a high-complexity center in Cali, Colombia. The medical records of patients with ANDs diagnosed by neurologists, who received at least one TPE in our institution between 2011 and 2018, were included in our analyses. In each case, the number of treatment cycles, type of replacement solution, clinical characteristics as duration of inpatient stay, adverse effects, and death of patients were evaluated. For identifying relapses, admissions due to ANDs up to one year after TPE were quantified.

TPE was prescribed by neurologists and was always administered in the intensive care unit (ICU) by trained nurses. The procedure comprised centrifugation, where prophylactic calcium was not routine. In each case, according to TPE characteristics (e.g., volume, replacement solution), anticoagulation with citrate and replacement solutions were administered in the proportions of $1: 12$ to 1 : 16.

Since most of the evaluated diagnoses (e.g., MG, GBS, and CIDP) have a disease-specific scale, we used the modified Rankin Scale (mRS) to measure and compare the clinical improvement between our patients. Although initially used for stroke treatment, it has also been applied in several different contexts including the evaluation of outcomes in NMOSD [12], AE [13, 14], MG [15], CIDP [16], GBS [17], and other neurological conditions [18].

Thus, the mRS was applied to every patient at three different times: at admission, using the admission note of the department of neurology; after TPE, using their progress note registered after completing all the cycles; and 90-days after receiving TPE, using medical records of follow-up appointments with neurologists. mRS measurement was performed by the research group with a previous training by a neurology resident.

The mRS was first introduced in 1957 for measuring outcomes in acute stroke and was modified to its sevengrade version as follows: 0 , no symptoms; 1 , no significant disability; 2, slight disability; 3 , moderate disability; 4 , moderately severe disability; 5 , severe disability; and 6 , dead $[19,20]$. The proposed interpretation of the mRS is based on two ranges of scores, $0-2$ and 3-6, which are defined as favorable and nonfavorable, respectively; however, when looking at scores as individual points, changes in a single one have been stated to be clinically relevant [21].

Statistical analyses were performed using STATA v.14 ${ }^{\circledR}$ (StataCorp LP, College Station, TX). Quantitative variables are shown as means and medians with standard deviations (SD) and interquartile ranges (IQRs) according to their distribution on the basis of the Shapiro-Wilk test. Quantitative variables are also shown with frequencies and proportions. mRS scores are presented as medians with IQRs; scores at admission and after TPE were compared with the Wilcoxon matched-pair signed-rank test.

\section{Results}

A total of 187 patients were included in this study. Most of them were women $(n=104,55.6 \%)$, and the median age at admission was 50 (IQR 32-64) years. The most frequent AND was MG, with 70 cases (37.4\%), followed by GBS, NMOSD, CIDP, and AE (Table 1).

TPE was performed using the centrifugation method, and a catheter was placed at the jugular $(n=166,88.7 \%)$, femoral $(n=18,9.6 \%)$, or subclavian $(n=3,1.6 \%)$ positions.

The replacement solutions used in TPE were albumin, fresh frozen plasma, and succinylated gelatin. Albumin alone was used in most patients $(n=131,70.5 \%)$, whereas albumin in combination with fresh frozen plasma was used in one patient only. In the general sample, the median number of TPEs per patient was five (IQR 5-5) (Table 1). Separated by diagnosis, it was the same in MG, AE, and CIDP; in NMOSD and GBS, the median remained five with a range slightly wider (IQR 5-6). All of the TPEs were indicated daily. The mean exchanged volume was $2278 \mathrm{~mL}$ (SD 525.7), and the mean TPE time was 67 minutes (SD 17.5).

Patients had a median of inpatient stay of 12 (IQR 8-19) days. Those with AE had the longest of all, with a median of 24.5 (IQR 17-49) days; this group also required more days in the ICU than other groups. The complications observed after TPE was provided were as follows: $106(56.6 \%)$ patients presented with hypotension, $102(54.4 \%)$ presented with electrolytic disorders, and 85 (45.4\%) presented with some complications associated with the Mahurkar catheter (other complications were found at a lesser extent). The presence of infection was evaluated during the 14 days following TPE: a total of $21(11.2 \%)$ cases were observed, of which the majority were patients with GBS $(n=10,47.6 \%)$. Infections were subdivided on the basis of the causative agent, with bacteria as the principal factor $(n=17 / 21,80.9 \%)$; viral infections occurred in two $(9.5 \%)$ patients, fungal infection in two (9.5\%), mycobacterial infection in one (4.8\%), and parasitic infection in one $(4.8 \%)$. The most common pathogen was $E$. coli, with 4 cases (19\%), followed by $P$. mirabilis and $S$. aureus, both with 3 cases (14.3\%). Two individuals had two agents of infection at the same time (Table 2).

Three (1.6\%) patients died during hospitalization due to causes associated with their neurological disease and septic 
TABle 1: Demographic and TPE characteristics.

\begin{tabular}{lc}
\hline Characteristics & $N(\%)$ \\
\hline Age $^{* *}$ & $50(32-64)$ \\
\hline Sex & \\
Female & $104(55.6)$ \\
Male & $83(44.4)$ \\
\hline Autoimmune neurological disease & $187(100)$ \\
MG & $70(37.4)$ \\
GBS & $53(28.3)$ \\
NMOSD & $35(18.7)$ \\
CIDP & $23(12.3)$ \\
AE & $6(3.2)$ \\
\hline Types of replacement solutions & \\
Albumin & 131 \\
Succinylated gelatin & 45 \\
Albumin + succinylated gelatin & 10 \\
Albumin + fresh frozen plasma & 1 \\
Number of TPEs by patient & \\
\end{tabular}

${ }^{* *}$ Median (IQR). MG, myasthenia gravis; GBS, Guillain-Barré syndrome; NMOSD, neuromyelitis optica spectrum disorders; CIDP, chronic inflammatory demyelinating polyneuropathy; $\mathrm{AE}$, autoimmune encephalitis. TPE, therapeutic plasmatic exchange.

shock. Clinical relapse in the year following TPE was measured, and the largest proportion of relapses occurred in patients with MG (Table 2).

On the basis of the mRS score for each patient at admission and after TPE, 99 (52.9\%) patients showed decreased scores after TPE, 67 (35.8\%) showed no difference, and $21(11.2 \%)$ showed increased scores after therapy. Although three patients died, two were receiving TPE at the time of death and the other one had finished TPE and remained alive for three additional months, which explains the two scores of " 6 " in the final measurement (Table 3). In addition, a statistically significant difference was noted between the median mRS scores before and after TPE in general $(p=0)$ and for each disease: MG, $p=0$; GBS, $p=0.0019$; NMOSD, $p=0.004$; AE, $p=0.0516$. CIDP was the only one in which the difference was not significant $(p=0.2019)$ (Table 4$)$.

We calculated the mean/median time patients took to present relapses depending on their $\mathrm{mRS}$ after TPE and 90 days after TPE. It is important to notice that only 104 patients have a calculated mRS 90 days after TPE due to absence of medical records in that specific time (Table 5).

On the other hand, we calculated the difference in points between the mRS before and after TPE (the "Delta TPE") and looked for any interrelation with relapse time, but we did not find any.

\section{Discussion}

This is a real-life study that shows the experience with the usage of TPE in multiple ANDs in a big cohort of 187 patients who were treated at a high-complexity center in Colombia. In 2019, the ASFA guidelines [1] defined specific recommendations for the use of TPE, and GBS, CIDP, MG, and NMOSD were included among the diseases of interest. Of these diseases, the strongest grades of recommendation were assigned to MG and CIDP, whereas the weakest grades were assigned to NMOSD and GBS [1]. Furthermore, neurological conditions in general are known to account for $44 \%$ of the cases in which TPE is indicated [22].

As previously discussed, TPE is as effective as IVIG in the treatment of GBS, CIDP, and MG; therefore, the decision to choose one or another treatment depends, in most cases, on the clinician's experience, treatment availability, and expenses. What can vary is the scheme in which the treatment is provided upon diagnosis [23]. In the present study, TPE was indicated in every patient due to the severity of their disease or therapeutic failure of other therapies, with MG being the most frequently observed disease (similar to previous reports) along with GBS [22]. In our center, TPE, rather than IVIG, is the preferred management option for ANDs, and it achieves favorable results.

Generally, the number of treatment cycles was approximately five, consistent with descriptions that report a total fluid exchange divided five times in MG [24], GBS [22], and CIDP [3, 25]. Jiao et al. reported a slightly different range, 2-7 treatment cycles, in 29 patients with NMOSD [26], and Moser et al. reported a wider range of cycles, 3-13, in 12 patients with AE [27].

In the analyses of the complications, hypotension occurred in approximately half of our patients even though a replacement solution was provided with TPE to prevent this outcome; this differs to apheresis where there is no fluid replacement [2]. Nonetheless, hypotension is common when using TPE for different indications [27-31]. Hydroelectrolytic disorders were the second-most frequent adverse reaction, not only in our study but also in the study by Clarck et al., who found that 59 of their patients required the replacement of at least one electrolyte after TPE (primarily for potassium and magnesium abnormalities) [30]. In our patients, $11.2 \%$ presented with infections, of which $80.9 \%$ were caused by bacteria. This finding is similar to a group studied by Lemaire et al., who reported infections caused primarily by bacteria, followed by viruses and fungus [29]. Severe adverse reactions, such as hypersensibility reactions and arrhythmias, were seen at a lower frequency in our group.

Lemaire et al. reported 50 patients who were admitted to the ICU, with $40 \%$ admitted due to neurological causes. In their cohort, the median inpatient stay was 20 (IQR 12-35) days, which was similar to our findings (20 days, IQR 8-19), whereas they reported a mortality proportion of $8 \%$ compared with $1.6 \%$ in our group [29]. As has been previously stated, two of the deaths in our study occurred before TPE was completed; thus, in some cases, these treatment options are given to critical patients in whom a clinical response may be difficult to achieve [30].

Clinical responses after TPE have been measured in different ways among several studies. For instance, Morgan et al. clinically evaluated the improvement in patients with $\mathrm{NMO}$, concluding that there was a moderate improvement in functional scales [32]. For MG, CIDP, and GBS, Momtaz et al. focused on muscle strength, finding that of 31 patients with MG, $83.8 \%$ achieved a complete response; of 8 patients with CIDP, $87.5 \%$ achieved such a response; and of 39 patients with GBS, $51.3 \%$ responded in this way [25]. 
TABLE 2: Outcomes related to TPE.

\begin{tabular}{|c|c|c|c|c|c|c|}
\hline & $\begin{array}{c}\text { General, } \\
n=187\end{array}$ & $\begin{array}{c}\text { MG, } \\
n=70\end{array}$ & $\begin{array}{c}\text { GBS } \\
, n=53\end{array}$ & $\begin{array}{c}\text { NMOSD, } \\
n=35\end{array}$ & $\begin{array}{l}\text { CIDP, } \\
n=23\end{array}$ & AE, $n=6$ \\
\hline \multicolumn{7}{|c|}{ Days of inpatient stay, median (IQR) } \\
\hline Inhospital stay & $12(8-19)$ & $11(7-15)$ & $13(9-33)$ & $12(8-18)$ & $10(7-16)$ & $24.5(17-49)$ \\
\hline ICU stay & $7(6-12)$ & $7(6-10)$ & $9(6-24)$ & $6(6-9)$ & $6(6-8)$ & $10(6-15)$ \\
\hline \multicolumn{7}{|l|}{ Complications, $n(\%)$} \\
\hline Hypotension & $106(56.6)$ & $35(50)$ & $35(66)$ & $17(48.5)$ & $15(65.2)$ & $4(66.6)$ \\
\hline Hydroelectrolytic disorders & $102(54.5)$ & $35(50)$ & $30(56.6)$ & $23(65.7)$ & $11(47.8)$ & $3(50)$ \\
\hline Related to Mahurkar catheter & $85(45.4)$ & $29(41.2)$ & $26(49)$ & $15(42.8)$ & $12(52.1)$ & $3(50)$ \\
\hline Bleeding & $56(29.9)$ & $22(31.4)$ & $19(35.8)$ & $9(25.7)$ & $4(17.3)$ & $2(33.3)$ \\
\hline Infections & $21(11.2)$ & $5(7.1)$ & $10(18.8)$ & $4(11.4)$ & $2(8.6)$ & 0 \\
\hline Thrombosis & $4(2.1)$ & $1(1.4)$ & $3(5.6)$ & 0 & 0 & 0 \\
\hline Hypersensibility & $2(1)$ & $1(1.4)$ & 0 & 0 & 0 & $1(16.6)$ \\
\hline Arrhythmias & $2(1)$ & $1(1.4)$ & 0 & $1(2.8)$ & 0 & 0 \\
\hline Hemolysis & 0 & 0 & 0 & 0 & 0 & 0 \\
\hline \multicolumn{7}{|c|}{ Death during hospitalization, $n(\%)$} \\
\hline & $3(1.6)$ & 0 & $1(1.8)$ & 0 & $2(8.6)$ & 0 \\
\hline \multicolumn{7}{|c|}{ Relapse during the year after, $n(\%)$} \\
\hline Number of patients & $59(31.5)$ & $33(47.1)$ & $3(5.6)$ & $14(40)$ & $7(30.4)$ & $2(33.3)$ \\
\hline
\end{tabular}

MG, myasthenia gravis; GBS, Guillain-Barré syndrome; NMOSD, neuromyelitis optica spectrum disorders; CIDP, chronic inflammatory demyelinating polyneuropathy; AE, autoimmune encephalitis IQR, interquartile range; ICU, intensive care unit.

TABLE 3: Number of patients in each category of the modified Rankin Scale before and after TPE.

\begin{tabular}{|c|c|c|c|c|c|c|c|}
\hline \multirow{2}{*}{ Score at admission } & \multicolumn{7}{|c|}{ Score after TPE } \\
\hline & 0 & 1 & 2 & 3 & 4 & 5 & 6 \\
\hline 1 & 5 & 13 & 7 & 1 & 1 & 0 & 0 \\
\hline 2 & 2 & 5 & 8 & 0 & 0 & 1 & 0 \\
\hline 3 & 3 & 4 & 20 & 15 & 4 & 2 & 0 \\
\hline 4 & 7 & 4 & 7 & 23 & 18 & 3 & 0 \\
\hline 5 & 1 & 2 & 0 & 12 & 4 & 13 & 2 \\
\hline
\end{tabular}

Each cell shows the number of patients who had a specific score at admission and after TPE. In green, patients who had a reduction in the scores after TPE; in blue, patients in whom the scores remained equal; in gray, patients who had an increase in the scores.

TABLE 4: Modified Rankin Scale scores before TPE compared with scores after TPE.

\begin{tabular}{lccr}
\hline Diagnosis** & Before TPE & After TPE & $p$ value \\
\hline General & $4(3-4)$ & $3(2-4)$ & $\mathbf{0}$ \\
MG & $3(2-4)$ & $1(1-2)$ & $\mathbf{0}$ \\
GBS & $4(3-5)$ & $3(3-4)$ & $\mathbf{0 . 0 0 1 9}$ \\
NMOSD & $4(3-4)$ & $3(2-4)$ & $\mathbf{0 . 0 0 4}$ \\
CIDP & $4(3-4)$ & $3(2-4)$ & 0.2019 \\
AE & $4(3-5)$ & $3(2-3)$ & $\mathbf{0 . 0 5 1 6}$ \\
\hline
\end{tabular}

${ }^{* *}$ Median (IQR), Wilcoxon matched-pairs signed-ranks test.

Titulaer et al. described a cohort of 577 patients diagnosed with $\mathrm{AE}$, of whom 461 received first-line therapies, including TPE: $97 \%$ maintained $\mathrm{mRS}$ scores of $0-2$ during a year of follow-up [33].

In this way, what we identify as the strength of our study is that we could compare multiple ANDs and bring objective data regarding clinical improvement after TPE with the application of the mRS, based on the experience of different authors who evaluated these diseases separately. It is to remark that although mRS is not a disease-specific scale for the diagnoses of interest, it is one that allows an approach in a more unified way among them. Then, similarly to the studies previously mentioned, $52.9 \%$ of our patients showed an improvement in their $\mathrm{mRS}$ scores whereas $35.8 \%$ did not show any response to treatment. Although no significant difference was noted between mRS scores before and after TPE in patients with CIDP, a decrease in median scores for each disease was observed in all diagnoses.

We did not find a pattern regarding time until relapses in relation to the $\mathrm{mRS}$ scores, but in the patients with a score of " 0 " at 90-days after TPE, it took the longest period of time to present a relapse, as would be expected. Then, it is reasonable to think that other factors may be associated with a better outcome following TPE, and it may be useful to consider 
TABLe 5: Time until relapses according to mRS scores after TPE and 90 days after TPE.

\begin{tabular}{|c|c|c|c|c|c|c|c|}
\hline \multicolumn{4}{|c|}{ Relapses $<90$ days after TPE } & \multicolumn{4}{|c|}{ Relapses $>90$ days after TPE } \\
\hline $\begin{array}{l}\text { mRS score } \\
\text { after TPE }\end{array}$ & $\begin{array}{l}\text { Patients with the } \\
\text { score }(n=185)\end{array}$ & $\begin{array}{l}\text { Patients who } \\
\text { relapsed, } n(\%)\end{array}$ & $\begin{array}{c}\text { Time until relapse } \\
\text { (days) }\end{array}$ & $\begin{array}{c}\text { mRS score } 90 \\
\text { days after } \\
\text { TPE }\end{array}$ & $\begin{array}{l}\text { Patients with the } \\
\text { score }(n=104)\end{array}$ & $\begin{array}{l}\text { Patients who } \\
\text { relapsed, } n(\%)\end{array}$ & $\begin{array}{l}\text { Time until } \\
\text { relapse (days) }\end{array}$ \\
\hline 5 & 19 & $1(5,55)$ & 71 & 5 & 1 & $1(100)$ & 195 \\
\hline 4 & 27 & $2(7,4)$ & $34,5(26-43)^{* *}$ & 4 & 15 & $4(26.7 \%)$ & $\begin{array}{c}139,5(115,25- \\
166)^{* *}\end{array}$ \\
\hline 3 & 51 & $8(15,7)$ & $\begin{array}{c}12,5 \\
(4,25-47,75)^{* *}\end{array}$ & 3 & 30 & $7(23 \%)$ & $151(29,35)^{*}$ \\
\hline 2 & 42 & $7(16,7)$ & $50(30,04)^{*}$ & 2 & 28 & $5(17,86 \%)$ & $160,2(69,79)^{*}$ \\
\hline 1 & 28 & $6(22,2)$ & $24,6(15,9)^{*}$ & 1 & 22 & $5(22,7 \%)$ & $201,2(94,73)^{*}$ \\
\hline 0 & 18 & $4(22,2)$ & $\begin{array}{c}16,5 \\
(4,75-56,75)^{* *}\end{array}$ & 0 & 8 & $1(12,5 \%)$ & 272 \\
\hline
\end{tabular}

${ }^{*}$ Mean (SD); ${ }^{* *}$ Median (IQR). mRS, modified Rankin Scale.

these when making treatment decisions or considering expectations. In NMOSD, these factors include anti-AQP4 seronegativity (patients who are seronegative for anti-AQP4 but seropositive for anti-myelin oligodendrocyte glycoprotein generally present better outcomes) [12] and combination treatments with immunosuppressants [34]. In GBS, additional factors include the number of exchanges on the basis of the severity of disease [35] and the patient's age [17]. An efficient diagnosis approach [14] and early therapy initiation are common characteristics in various studies $[12,14,33,35,36]$.

\section{Conclusion}

We showed TPE to have a high tolerance and strong safety profile in various ANDs. Furthermore, the improvement in mRS scores reflects the effectiveness of TPE in the treatment of MG, GBS, NMOSD, and AE.

\section{Study Limitations}

This study was retrospective in nature, which is why the measurements of clinical response had to be evaluated using a nonspecific scale. Furthermore, the scores used depend on information that was registered on medical records, which may lower the accuracy of the scores.

Additionally, the number of patients in each diagnosis limits the comparison between ANDs. The small number of patients with AE must be considered when interpreting the results. Finally, not every patient went for outpatient control visit 90-days after TPE, diminishing the study sample at that time, thus limiting the evaluation and/or comparison of variables such as mRS 90 days after TPE.

\section{Data Availability}

The data used to support the findings of this study are available from the corresponding author upon request.

\section{Disclosure}

This work has been accepted for oral abstract presentation in the 12th International Congress on Autoimmunity.

\section{Conflicts of Interest}

The authors declare that they have no conflicts of interest.

\section{Acknowledgments}

The authors thank Andrés M. Castro, from Center of Clinical Research at Fundación Valle del Lili, for the statistical analysis.

\section{References}

[1] A. Padmanabhan, L. Connelly-Smith, N. Aqui et al., "Guidelines on the use of therapeutic apheresis in clinical practice-evidence-based approach from the writing committee of the American society for apheresis: the eighth special issue," Journal of Clinical Apheresis, vol. 34, no. 3, pp. 171-354, 2019.

[2] H. M. Reeves and J. L. Winters, "The mechanisms of action of plasma exchange," British Journal of Haematology, vol. 164, no. 3, pp. 342-351, 2014.

[3] E. Kaya, M. Keklik, M. Şencan et al., "Therapeutic plasma exchange in patients with neurological diseases: multicenter retrospective analysis," Transfusion and Apheresis Science, vol. 48, no. 3, pp. 349-352, 2013.

[4] A. L. Oaklander, M. P. Lunn, R. A. Hughes, I. N. van Schaik, C. Frost, and C. H. Chalk, "Treatments for chronic inflammatory demyelinating polyradiculoneuropathy (CIDP): an overview of systematic reviews," Cochrane Database of Systematic Reviews, vol. 1, Article ID CD010369, 2017.

[5] S. Chevret, R. A. Hughes, and D. Annane, "Plasma exchange for Guillain-Barré syndrome," Cochrane Database of Systematic Reviews, vol. 2, Article ID CD001798, 2017.

[6] M. Bonnan, R. Valentino, S. Debeugny et al., "Short delay to initiate plasma exchange is the strongest predictor of outcome in severe attacks of NMO spectrum disorders," Journal of Neurology, Neurosurgery \& Psychiatry, vol. 89, no. 4, pp. 346-351, 2018.

[7] D. Barth, M. Nabavi Nouri, E. Ng, P. Nwe, and V. Bril, "Comparison of IVIg and PLEX in patients with myasthenia gravis," Neurology, vol. 76, no. 23, pp. 2017-2023, 2011.

[8] A. McGrogan, G. C. Madle, H. E. Seaman, and C. S. de Vries, "The epidemiology of Guillain-Barré syndrome worldwide. A systematic literature review," Neuroepidemiology, vol. 32, no. 2, pp. 150-163, 2009. 
[9] D. I. Jonsson, O. Sveinsson, R. Hakim, and L. Brundin, "Epidemiology of NMOSD in Sweden from 1987 to 2013," Neurology, vol. 93, no. 2, pp. e181-e189, 2019.

[10] I. Casetta, E. Groppo, R. Gennaro et al., "Myasthenia gravis: a changing pattern of incidence," Journal of Neurology, vol. 257, pp. 2015-2019, 2010.

[11] D. Dubey, S. J. Pittock, C. R. Kelly et al., "Autoimmune encephalitis epidemiology and a comparison to infectious encephalitis," Annals of Neurology, vol. 83, no. 1, pp. 166-177, 2018.

[12] Z. M. Ong, J. P. Schee, and S. Viswanathan, "Therapeutic plasma exchange (TPE) in neuromyelitis optica spectrum disorders (NMOSD) and related disorders in resource limited settings: outcomes in a multi-ethnic single center population ong," Therapeutic Apheresis and Dialysis, vol. 24, no. 3, pp. 312-323, 2019.

[13] X. Xu, Q. Lu, Y. Huang et al., "Anti-NMDAR encephalitis," Neurol Neuroimmunol Neuroinflammation, vol. 7, no. 1, p. e633, 2020.

[14] M. Nosadini, S. S. Mohammad, S. Ramanathan, F. Brilot, and R. C. Dale, "Immune therapy in autoimmune encephalitis: a systematic review," Expert Review of Neurotherapeutics, vol. 15, no. 12, pp. 1391-1419, 2015.

[15] M. E. Farrugia, C. Carmichael, B. J. Cupka et al., "The modified rankin scale to assess disability in myasthenia gravis: comparing with other tools," Muscle and Nerve, vol. 50, no. 4, pp. 501-507, 2014.

[16] E. Spina, A. Topa, R. Iodice et al., "Early predictive factors of disability in CIDP," Journal of Neurology, vol. 264, no. 9, pp. 1939-1944, 2017.

[17] S. H. Siddiqui, T. H. Siddiqui, M. U. Babar, A. Khoja, and S. Khan, "Outcomes of patients with Guillain Barre Syndrome-experience from a tertiary care hospital of a developing Asian country and review of regional literature," Journal of Clinical Neuroscience, vol. 62, pp. 195-198, 2019.

[18] P. Nihar, V. A. Rao, E. R. Heilman-Espinoza, R. Lai, R. A. Quesada, and A. C. Flint, "Simple and reliable determination of the modified rankin scale score in neurosurgical and neurological patients: the mRS-9Q," Neurosurgery, vol. 71, no. 5, pp. 971-975, 2012.

[19] J. P. Broderick, O. Adeoeye, and J. Elm, "The evolution of the modified rankin scale and its use in future stroke trials," Stroke, vol. 48, no. 7, pp. 2007-2012, 2017.

[20] B. Farrell, J. Godwin, S. Richards, and C. Warlow, "The United Kingdom transient ischaemic attack (UK-TIA) aspirin trial: final results," Journal of Neurology, Neurosurgery \& Psychiatry, vol. 54, no. 12, pp. 1044-1054, 1951.

[21] J. K. Harrison, K. S. McArthur, and T. J. Quinn, "Assessment scales in stroke: clinimetric and clinical considerations," Clinical Interventions in Aging, vol. 8, pp. 201-211, 2013.

[22] K. Gwathmey, R. A. Balogun, and T. Burns, "Neurologic indications for therapeutic plasma exchange: an update," Journal of Clinical Apheresis, vol. 26, no. 5, pp. 261-268, 2011.

[23] M. Fernández-Zarzoso, I. Gómez-Seguí, and J. de la Rubia, "Therapeutic plasma exchange: review of current indications," Transfusion and Apheresis Science, vol. 58, no. 3, pp. 247-253, 2019.

[24] H. Ebadi, D. Barth, and V. Bril, "Safety of plasma exchange therapy in patients with myasthenia gravis," Muscle and Nerve, vol. 47, no. 4, pp. 510-514, 2013.

[25] M. Momtaz, A. Fayed, K. Marzouk, and A. Shaker, "Therapeutic plasma exchange outcomes in cairo university hospitals: 6 years experience," Therapeutic Apheresis and Dialysis, vol. 22, no. 6, pp. 666-673, 2018.
[26] Y. Jiao, L. Cui, W. Zhang et al., "Plasma exchange for neuromyelitis optica spectrum disorders in Chinese patients and factors predictive of short-term outcome," Clinical Therapeutics, vol. 40, no. 4, pp. 603-612, 2018.

[27] T. Moser, G. Harutyunyan, A. Karamyan et al., “Therapeutic plasma exchange in multiple sclerosis and autoimmune encephalitis: a comparative study of indication, efficacy and safety," Brain Sciences, vol. 9, no. 10, pp. 1-11, 2019.

[28] D. Aguirre-Valencia, J. Naranjo-Escobar, I. Posso-Osorio et al., "Therapeutic plasma exchange as management of complicated systemic lupus erythematosus and other autoimmune diseases," Autoimmune Diseases, vol. 2019, pp. 1-10, 2019.

[29] A. Lemaire, N. Parquet, L. Galicier et al., "Plasma exchange in the intensive care unit: technical aspects and complications," Journal of Clinical Apheresis, vol. 32, no. 6, pp. 405-412, 2017.

[30] S. L. Clark and A. A. Rabinstein, "Safety of intravenous immunoglobulin and plasma exchange in critically ill patients," Neurological Research, vol. 37, no. 7, pp. 593-598, 2015.

[31] I. Kleyman and T. H. Brannagan, "Treatment of chronic inflammatory demyelinating polyneuropathy," Current Neurology and Neuroscience Reports, vol. 15, no. 7, p. 47, 2015.

[32] S. M. Morgan, N. D. Zantek, and A. F. Carpenter, "Therapeutic plasma exchange in neuromyelitis optica: a case series," Journal of Clinical Apheresis, vol. 29, no. 3, pp. 171-177, 2014.

[33] M. J. Titulaer, L. McCracken, I. Gabilondo et al., "Treatment and prognostic factors for long-term outcome in patients with anti-N-Methyl-D-Aspartate (NMDA) receptor encephalitis: a cohort study," The Lancet Neurology, vol. 12, no. 2, pp. 157-165, 2013.

[34] M. Netravathi, B. Hari krishna, B. Maya Dattatraya et al., "Clinical, neuroimaging and therapeutic response in AQP4positive NMO patients from India," Multiple Sclerosis and Related Disorders, vol. 30, pp. 85-93, 2019.

[35] S. Liu, C. Dong, and E. E. Ubogu, "Immunotherapy of Guillain-Barré syndrome," Human Vaccines \& Immunotherapeutics, vol. 14, pp. 2568-2579, 2018.

[36] P. Codron, M. Cousin, J.-F. Subra et al., "Therapeutic plasma exchange in chronic dysimmune peripheral neuropathies: a 10-year retrospective study," Journal of Clinical Apheresis, vol. 32, no. 6, pp. 413-422, 2017. 